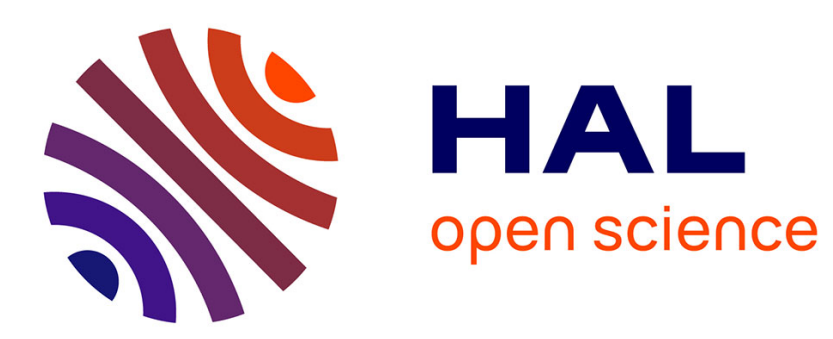

\title{
Characterization of mesoscale instabilities in localized granular shear using digital image correlation
}

Amy L Rechenmacher, Sara Abedi, Olivier Chupin, Andrés D Orlando

\section{To cite this version:}

Amy L Rechenmacher, Sara Abedi, Olivier Chupin, Andrés D Orlando. Characterization of mesoscale instabilities in localized granular shear using digital image correlation. Acta Geotechnica, 2011, 6 (4), pp 205-217. 10.1007/s11440-011-0147-2 . hal-00972573

\section{HAL Id: hal-00972573 https://hal.science/hal-00972573}

Submitted on 13 Dec 2017

HAL is a multi-disciplinary open access archive for the deposit and dissemination of scientific research documents, whether they are published or not. The documents may come from teaching and research institutions in France or abroad, or from public or private research centers.
L'archive ouverte pluridisciplinaire HAL, est destinée au dépôt et à la diffusion de documents scientifiques de niveau recherche, publiés ou non, émanant des établissements d'enseignement et de recherche français ou étrangers, des laboratoires publics ou privés. 


\title{
Characterization of mesoscale instabilities in localized granular shear using digital image correlation
}

\author{
Amy L. Rechenmacher · Sara Abedi • \\ Olivier Chupin · Andrés D. Orlando
}

\begin{abstract}
Within shear bands in sands, deformation is largely non-affine, stemming primarily from buckling of well-known force chains and also from vortex-like structures. In the spirit of current trends toward multiscale modeling, understanding the links between these mesoscale deformational entities and corresponding macroscale response will form the basis for the next generation of sand behavioral models and may also aid in efforts to understand jamming-unjamming transitions in dense granular flows in general. Experimental methods to quantify and characterize such subscale kinematics, in particular in real sands, will play critical roles in these efforts. Digital Image Correlation (DIC) is a fast growing experimental technique to nondestructively measure surface displacements from digital images. Here, DIC has been employed to identify and characterize the development of vortex structures inside shear bands formed in dense sands during plane strain compression. A rigorous assessment of the DIC method has been performed, in particular for subscale behavioral characterization in unbonded granular solids, and guidelines are offered for accurate implementation. While DIC systematically overestimates shear band thickness, a methodology has been devised to compensate for
\end{abstract}

Paper presented at the International Workshop on Multiscale \& Multiphysics Processes in Geomechanics, June 23-25, 2010, Stanford University.

A. L. Rechenmacher $(\bowtie) \cdot$ S. Abedi · A. D. Orlando Department of Civil Engineering, University of Southern California, 3620 S. Vermont Ave, Los Angeles, CA 90089, USA e-mail: arechenm@usc.edu

O. Chupin

LUNAM Université, IFSTTAR, IM,

Structures, 44341 Bouguenais, France this overestimation. Shear band thickness for four different uniform sands were found to range between 6 and 9 grain diameters, and for a well-graded sand between 8 and 9.5 grain diameters. These determinations agree with visual inspections of grain kinematics from the image data, as well as recent theoretical predictions.

Keywords Digital image correlation - Force chain . Granular materials $\cdot$ Length scale $\cdot$ Sand $\cdot$ Shear band . Vortex

\section{Introduction}

In dense sands, failure is ubiquitously associated with the formation of shear bands, which are geometrically constrained zones comprising intense, predominantly shearlike deformation. Shear bands in sands are of considerable thickness, with values reported on the scale of about 8-25 grain diameters, e.g., [2, 3, 14, 21, 30, 34]. The displacement field within the shear band is of particular interest, as once the shear band forms, all significant material straining is confined only to within the shear band, e.g., [30]: subscale kinematics within this material zone then govern macroscopic stress evolution from softening to critical state.

Within shear bands in dense granular flows, deformation is largely non-affine [16, 17, 24, 25, 29, 31]. This nonaffinity stems primarily from the buildup and collapse of "force chains", which are finite-sized, quasi-linear, chainlike particle groups transmitting above-average contact forces [11, 22, 42], and also coherent vortex-like structures or "circulation cells" [1, 41, 43, 48, 49]. Since force chains and vortices ostensibly play a strong role in granular material deformation, an understanding of their behavioral 
evolution represents a fundamental goal of granular mechanics. Current knowledge about the appearance and behavior of vortex-like structures in particular has been derived largely from particle-based numerical simulations or experiments on spherical or circular particles, e.g., [10, 45]. Experimental work on real sands has been limited. Cross sections of epoxy-hardened sand specimens have been investigated microscopically [12, 18, 25], and more recently, micro-X-ray Computed Tomography $(\mu \mathrm{CT})$ has been used, e.g., $[15,40]$. Both techniques allow for very detailed particle contact maps. However, kinematic data are not obtained, in particular over the relatively short strain increments thought to comprise the lifetimes of vortex-like structures (e.g., [45]).

The experimental technique of Digital Image Correlation (DIC) has gained widespread acceptance as a reliable method for non-contact measurement of full field surface displacements. DIC considers subsets of pixels with unique gray-level patterns and avails displacement measurement by mapping these subsets between two images capturing a deformation process. DIC is particularly powerful when the displacement and/or strain field is heterogeneous, wherein conventional boundary-based sensing techniques fail to capture true material straining. DIC has been successfully applied in a wide range of materials [47], including sands, but primarily for the analysis of macroscopic shear band properties, such as inclination and patterning, e.g., [20, 30, $33,36,50]$. In shear bands in bonded solids, wherein the displacement field is discontinuous, a main issue is DIC non-convergence in the vicinity of the displacement jump, e.g., [27, 32]. In sands, however, the displacement field in and around the shear band is continuous, so DIC is potentially applicable to capture "mesoscale" (the scale of a small cluster of grains) deformation inside shear bands. However, special considerations are warranted. First, for sands, material strain advances largely from inter-particle movement; as such, the grains themselves do not strain, potentially impacting DIC mapping accuracy. Note, however, that grain imperfections in even the most rounded of sands leads to moment resistance between grains, leading to coordinated motion, i.e., a grain moves in conjunction with its neighbors [5, 46]. Given this, grain clusters should tend to remain largely intact over small strain increments, so that such grain clusters represented within pixel subsets can potentially be tracked. A second issue relates to the choice of subset size. Subset size is generally balanced between being large enough to encompass a unique and identifiable gray-level intensity pattern [35], and small enough such that the deformation field is accurately approximated by the underlying shape function, e.g., [19, 26]. An additional factor is that the subset must be appropriately sized to capture the scale of the behavior of interest [6]: here, small enough to discern the kinematics of individual vortex structures, the scale of which are currently not known with certainty. As will be seen below, consideration and determination of this internal length scale is a controlling factor in the choice of subset size.

After much experience using DIC to characterize strain localization in sands, we have derived improved understanding of the technique and its limitations and offer here revised recommendations from previous works [30, 31] for its implementation, in particular for characterizing mesoscale deformations and kinematics inside shear bands. As an example of the power of DIC, we show here how DIC has enabled detection of vortex structures within shear bands. As digital cameras become more affordable, and DIC use in micro- and mesoscale soil behavioral characterization becomes more widespread, our findings will provide guidance for further DIC use, not just in sand, but in unbonded, granular solids in general. In the spirit of current trends toward multiscale modeling, understanding the links between micro-, meso-, and macroscale soil behavior will form the basis for the next generation of soil behavioral models, e.g., [4, 7], and grain-scale experimental techniques, such as DIC and $\mu \mathrm{CT}$, will play critical roles in these efforts. We first discuss the plane strain testing apparatus and experimental materials and procedures. We then discuss DIC use, specifically focusing on analysis parameters and insights for maximum subset size and limiting strain increments for analysis. We present an example of the typical spatial variation in kinematics along a shear band at the softening-critical state transition and show how previously observed patterns [29, 31] tie in with the formation of coherent vortex-like structures. Finally, we present a methodology for quantifying shear band thickness as a function of grain size using DIC. We note that previous estimates of shear band thickness using DIC [30] represented over-approximations. Revised estimates of previous results agree with current findings, and these new thickness estimates have been validated by the image data and recent theoretical predictions [44].

\section{Experimental methods and materials}

Specimens of saturated sand are tested in plane strain compression. The specimen configuration is conceptually depicted in Fig. 1. A $140-$ by $80-$ by $40-\mathrm{mm}$ (nominal dimensions) specimen is sealed inside a pliable, translucent latex membrane, and mounted between two glass-lined, acrylic sidewalls that inhibit strain in the out-of-plane direction (Fig. 1). The specimen-sidewall interfaces are lubricated with silicon oil to minimize in-plane frictional loss. The top and bottom platens (only bottom platen is shown in Fig. 1) also are glass-lined and lubricated to minimize boundary friction. The specimen base platen rests 


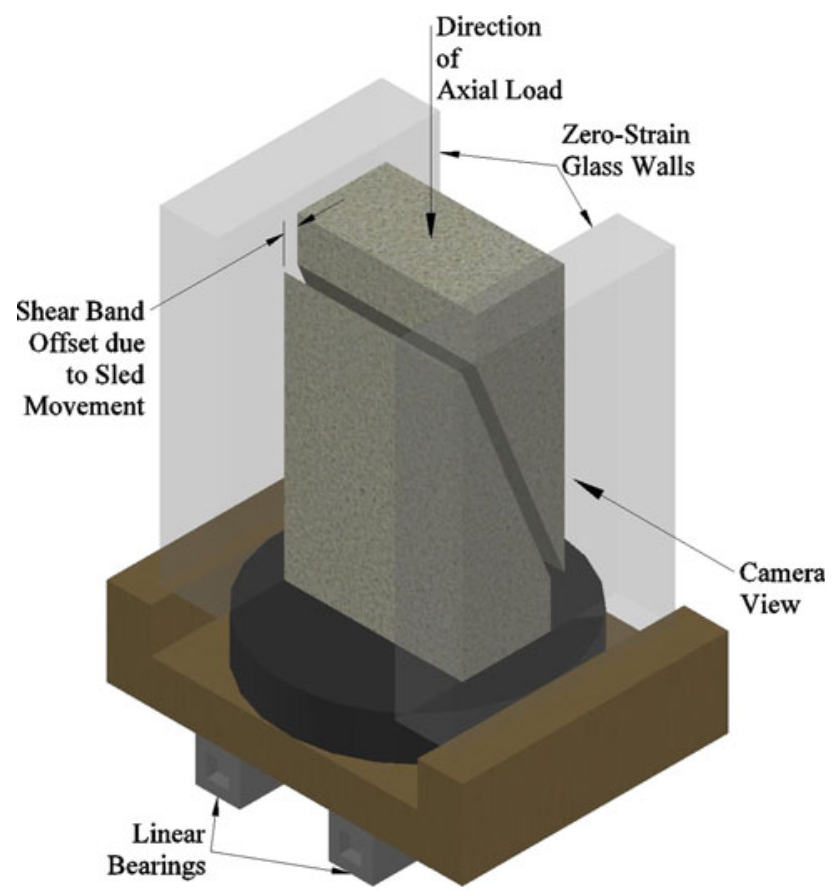

Fig. 1 Conceptual representation of plane strain test specimen and surrounding hardware

on a low-friction, linear bearing "sled", which permits the lateral offset required for unconstrained growth of a single shear band (Fig. 1). Load cells are mounted both above the top and below the bottom platens, the former to enable axial stress calculation and the latter to track frictional loss along the sidewall (friction angle losses are typically around 1-7 degrees). Additional load cells are mounted inside the back sidewall to measure out-of-plane force for intermediate principal stress calculation. LVDT displacement transducers are arranged around the specimen to measure macroscopic specimen axial and in-plane deformation and sled movement.

The specimen, sidewalls, and sled are sealed inside a $41 \mathrm{~cm}$ outer diameter, $3.175-\mathrm{cm}$-thick acrylic cylindrical cell (the large radius of which has a minimial effect on image distortion across the narrow specimen width [23]). The cell is filled with silicon oil, which provides the medium for minor principal stress application. Specimens are then saturated, consolidated anisotropically, and then sheared in drained, plane strain compression at a constant axial strain rate, typically $2 \%$ per hour.

The sands tested represented mixes of several different kinds of sand to achieve varied grain coloring to optimize DIC subset matching. Relevant properties for these sands are given in Table 1. The first, Concrete (C) sand, is manufactured or quarried. This sand is considered "well graded", and its grain sizes varied over more than an order of magnitude (from $2 \mathrm{~mm}$ down to around $0.1 \mathrm{~mm}$ ). The second sand, Masonry-Coarse (MC) sand, is a uniform
Table 1 Properties of sands

\begin{tabular}{llll}
\hline & $\begin{array}{l}\text { Concrete } \\
(\mathrm{C})\end{array}$ & $\begin{array}{l}\text { Masonry-Coarse } \\
(\mathrm{MC})\end{array}$ & $\begin{array}{l}\text { Silica-Coarse } \\
(\mathrm{SC})\end{array}$ \\
\hline$D_{50(\mathrm{~mm})}$ & 0.62 & 0.84 & 0.42 \\
$\mathrm{C}_{\mathrm{u}}\left(D_{60} / D_{10}\right)$ & 3.8 & 1.2 & 1.2 \\
$\mathrm{C}_{\mathrm{c}}\left(D_{30}^{2} /\left[D_{10} \times D_{60}\right]\right)$ & 0.67 & 1.1 & 1.0 \\
Specific gravity, $G_{\mathrm{s}}$ & 2.73 & 2.64 & 2.64 \\
\hline
\end{tabular}

$D_{\mathrm{XX}}$ represents the grain diameter for which $\mathrm{XX} \%$ of the sand by mass is smaller than this size

Table 2 Plane strain test data

\begin{tabular}{ll}
\hline Test No. ${ }^{\mathrm{a}}$ & $\mathrm{p}_{\mathrm{c}}{ }^{\prime}(\mathrm{kPa})^{\mathrm{b}}$ \\
\hline $\mathrm{C} 24$ & 107 \\
$\mathrm{MC} 21$ & 175 \\
$\mathrm{MC} 24$ & 237 \\
$\mathrm{MC} 35$ & 171 \\
SC10 & 129 \\
\hline
\end{tabular}

a All MC and SC specimens prepared at a relative densities $\left(D_{\mathrm{r}}\right) 90$ to $100 \%$; for $\mathrm{C} 24, D_{\mathrm{r}}=52 \%$

${ }^{\mathrm{b}} p_{c}^{\prime}=1 / 3\left(\sigma_{1}^{\prime}+\sigma_{2}^{\prime}+\sigma_{3}^{\prime}\right)$ mean normal effective stress at end of consolidation

sand and comprises a $90-10 \%$ mixture by mass, respectively, of Masonry sand (used in brick masonry mortar mixes and is also manufactured/quarried) and a Coarse concrete sand (also used for concrete mixes but from a different source as the Concrete sand above). The third sand, Silica-Coarse (SC) sand, is comprised of a 50-50\% mixture by mass of mined Silica sand and the same Coarse sand as in the MC sand. The sands comprising both MC and SC sands are sieved prior to mixing, to achieve the targeted median grain size given in Table 1. Grains for all sands are subrounded to subangular in shape.

To prepare specimens, dry sand was rained free-fall in air from a screened outlet in a deposition device sized slightly smaller than the specimen cross section. The deposition device was slowly raised throughout deposition so that the distance from the deposition outlet to the sand surface remained constant, leading to uniform bulk density throughout the specimen height. The top surface of the sand specimen was unobtrusively leveled before placement of the top platen, to avoid local material densification, and hence material nonuniformity. Nearly, four dozen tests have been performed between the current test program and a previous program $[13,30]$. Relevant parameters for the tests discussed herein are given in Table 2.

Throughout shear, digital images were collected at frequent intervals. Two different cameras were used for the SC and MC sand tests: a 4-Megapixel Q-Imaging PMI 4201 and a 5-Megapixel Point Grey Grasshopper. 


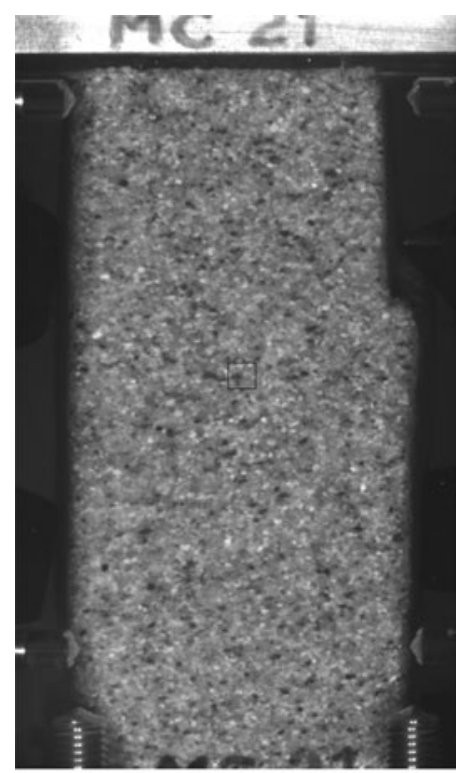

(a)

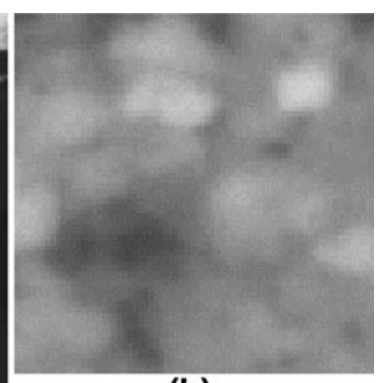

(b)

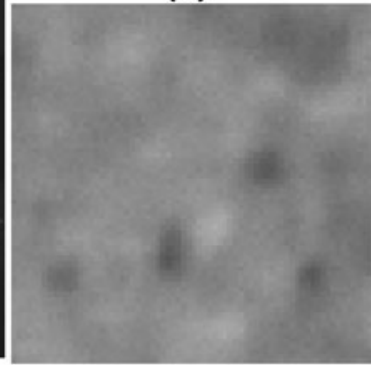

(c)
Fig. 2 Typical digital images: a MC sand specimen (test MC21); b gray-level variation within the $70 \times 70$ pixel subset indicated by the rectangle in a; c $70 \times 70$ pixel subset within an SC sand specimen

Figure 2a shows an image of a typical MC sand test specimen (test MC21). In this test, the shear band extends lower left to upper right (specimen offsets due to sled movement are seen just behind the screw on the lower left side and at about the two-thirds height on the right-hand side). A $70 \times 70$ pixel portion of the specimen is shown in Fig. 2b. The image scales are typically about $0.06 \mathrm{~mm} /$ pixel, so a $0.84-\mathrm{mm}$ sand grain occupies on average about 14 pixels. For comparative purposes, a $70 \times 70$ pixel subset of an SC sand specimen is shown in Fig. 2c. The $0.42-\mathrm{mm}$ sand grains here are about 7 pixels across. Graylevel histograms were typically unimodal with normal distributions. Cross hairs etched on the glass confining sidewall (barely visible just off the specimen edges of Fig. 2a) provide the basis for physical scale. The Concrete sand was tested in an earlier experimental phase, wherein a 1-Megapixel Kodak Megaplus 4.2 camera was used. Grain sizes for this well-graded sand ranged from about 4 to as large as 20 pixels. Note, all digital cameras used in our research are $\mathrm{B} / \mathrm{W}$ scientific grade, as they tend to yield less noise and higher reliability than consumer cameras.

\section{Digital image correlation (DIC)}

\subsection{Overview and typical results}

Digital Image Correlation (DIC) is a non-invasive experimental technique to measure surface displacements on a deforming material by matching reference pixel subsets in an initial image state with target subsets in an image of the deformed state. Full-field displacement information is obtained by tracking the motion of overlapping pixel subsets before and after deformation. The details of the DIC technique are well known and will not be described here. The reader is referred to Sutton et al. [37-39] and Bruck et al. [8] for more detail on this technique. The program VIC-2D by Correlated Solutions, Inc., was used to conduct all DIC analyses herein.

The following parameters were used to conduct the DIC analyses. Cubic spline interpolation (optimized 8 tap) was used for sub-pixel intensity interpolation (necessary for subpixel displacement resolution). As seen in Fig. 2b, c, black/white transitions typically were not abrupt, so application of a low-pass filter was found not to be necessary. Affine shape functions were used to approximate subset strain. The Zero-Mean Normalized Sum of Squared Differences (ZNSSD) criterion [39] was used as the error measure for subset matching. Subset size varied depending on the sand tested and will be discussed in more detail below. Subset center-to-center spacing was chosen to provide ensuing displacement data point spacing on the order of the size of a sand grain. DICs were typically performed over rectangular areas of interest (AOIs), capturing about $65-70 \%$ of the central portion of the imaged specimen face (avoiding hardware, shadows, etc.). The output of each DIC analysis was a rectangular grid of displacement data points, with data point locations corresponding to initial subset center positions.

Thus, each DIC data point represents the average displacement of the corresponding pixel subset, reflected at the subset's center. If material strain is locally uniform, and thus deformation is affine, then the subset center displacement will precisely correspond to the actual displacement of the material point physically represented at the subset's center. This correspondence between DICdetermined and actual material point displacements is explained in Fig. 3. Figure 3a depicts an idealized pixel subset (solid square box) capturing a group of three-bythree idealized grains. Ideally, the DIC measurement should reflect the displacement of the center grain, which here represents the material point at the subset center (note, in practice, a subset center will likely not systemically coincide with a grain location). Figure $3 \mathrm{~b}-\mathrm{e}$ represents hypothetical deformation scenarios, in which the solid circles represent grains that remain stationary during the deformation cycle and dotted circles represent grains that have moved (relative to the arrangement in Fig. 3a). The dashed lines surrounding the grains represent a rough approximation of affine subset strain, and the solid "dot" approximates the corresponding deformed subset center position (i.e., the DIC result). 
(a)

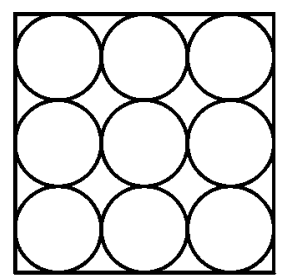

(b)

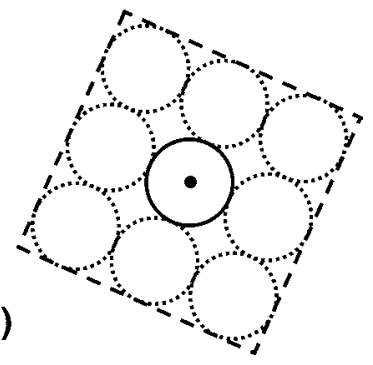

(c)

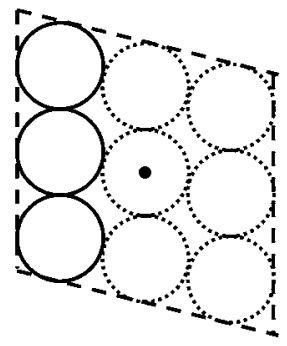

(d)

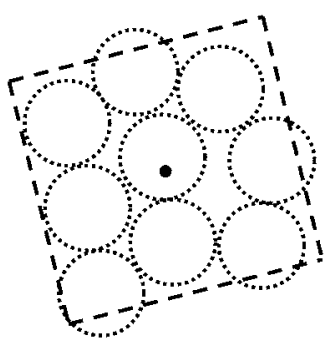

(e)

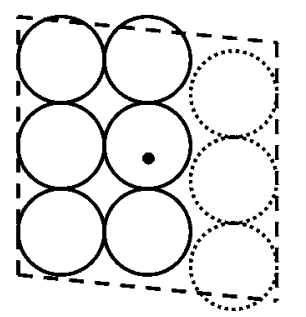

Fig. 3 Idealized grain deformation scenarios illustrating the nature of correspondences between material point and subset center displacements

Figure $3 b$, c represents two different cases of affine grain deformation: in Fig. $3 \mathrm{~b}$, the grains have rotated as a rigid body about the center grain, and in Fig. 3c, the two right columns of grains have sheared downward relative to the stationary left column. In both cases, the center of the deformed pixel subset exactly coincides with the displacement of the center grain. Figure $3 d$, e, on the other hand, illustrates two scenarios of non-affine grain deformation: in Fig. 3d, all grains have shifted non-uniformly relative to one another, and in Fig. 3e, only the right-most grain column has translated vertically downward. In both cases, the subset center point does not align with the deformed center grain position. Thus, amidst non-affine deformation, DIC incurs bias in the estimated, local displacement, the strength of which depends on the degree to which deformation is non-affine. However, this inaccuracy can be minimized or eliminated by conducting DIC analyses over small strain increments, as will be discussed below.

Figure $3 \mathrm{e}$ is reminiscent of a displacement field discontinuity such as would be the case for slip "microbands", which are thin zones of discrete slip, one to a few grains wide and several grains long. It is keen to envision that as one moves laterally away from the region of discrete slip, the subsets will eventually no longer be biased by the nonaffine deformation reflected in the slip. As a specific example, consider Fig. 3e to instead represent the case of a vertically oriented microband, where the material to the left of the band is moving uniformly upward and the material to the right of the band translates uniformly downward. From left to right across the microband, DIC measurements would be represented by a gradual transition of displacement vectors oriented straight up to straight down, with zero displacement for subsets centered on the slip and displacement vectors remaining parallel on either side of the transition. The width of the transition zone will depend on subset size: the smaller the subset, the narrower the transition zone. Thus, DIC will represent such discrete displacement boundaries as gradual transitions. Consideration of this effect will be needed in investigating the presence of microbands in our data.

Figure 4 shows a typical DIC result performed over a $0.2 \%$ axial strain increment in MC sand test (test MC 35) after a persistent shear band had fully formed. For this analysis, subset sizes were 73 pixels, spaced 13 pixels center to center (the choices of these parameters will be discussed below). Scaled displacement vectors for about every 25 th of the roughly 5000 data points across the AOI are shown to indicate relative direction and magnitude of the local displacements. Two different reference frames are used in the discussions that follow. Figure 4 a shows displacements observed from the camera reference frame: the sand material above the shear band is translating rigidly downward with the prescribed global axial displacement, the material below the shear band is translating laterally with the bearing sled, and all significant material strain is confined within the shear band (note that the shear band is "hitting" the top corner of the AOI and not the top platen). Alternatively, by subtracting out the base sled movements from the original displacement field, we reference displacements to a stationary base. In this "sled reference frame," shown in Fig. 4b, shear band displacement vectors 


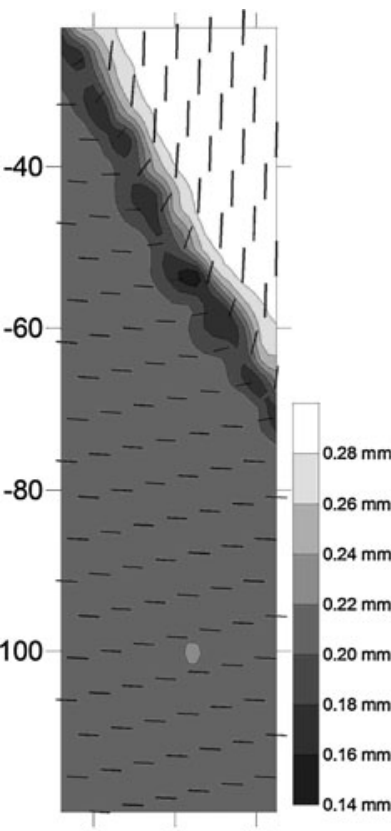

50

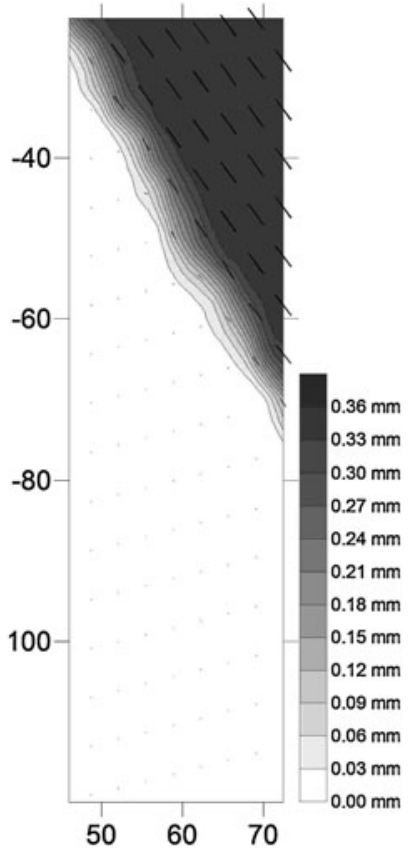

(b)
Fig. 4 Typical DIC result: a camera reference frame, b sled reference frame. Scaled displacement vectors for a portion of the subsets are shown for clarity

are largely parallel to the shear band axis and do not vary spatially along the shear band to the extent seen in the camera reference frame. The low displacement "islands" in Fig. 4a thus arise from fluctuations in magnitude of the displacement components perpendicular to the shear band $[29,31]$. As will be seen below, these fluctuations arise in part from upward to downward displacements on the rear and forward sides of coherent, vortex-like structures.

Note that due to the biasing effects at discrete behavioral transitions discussed above, DIC displacement results near the edges of the shear band, where the strain field is discontinuous, will be slightly biased. Strain magnitudes in these regions calculated from these DIC results then will necessarily be qualitative. However, as described above, the qualitative character of the displacement fields will still be representative. Moreover, nearer to the center of the shear band width, the spatial and temporal evolution of our distinguishing kinematics can indeed be precisely captured using DIC.

Occasionally, out-of-plane motion was observed in the form of: a) a sand grain "wiggling", manifesting local changes in gray-level intensity; and b) a sand grain "emerging" or "disappearing" into or out of the plane of the glass wall. Indeed, such occurrences locally compromised DIC correlation integrity. Fortunately, such motions were relatively infrequent. However, given that subsets were sized much larger than individual sand grains, the ensuing effects were merely local and had limited effect on observed behavior. Displacement measurement accuracy is roughly $\pm 0.009 \mathrm{~mm}[31]$.

\subsection{Subset size}

To enable characterization of distinct mesoscale kinematics associated with individual vortex structures, pixel subsets must be sized smaller than the anticipated size and spacing of these structures. Since the vortex structures of interest here are necessarily confined to within the shear band, our starting point is to size subsets no larger than to fit entirely within the shear band thickness.

For a shear band of physical thickness $t$, and inclination $\beta$, the limiting physical size of the subset, $l$, to fit entirely within the shear band is determined as shown in Fig. 5a. A problem arises, however, in the accurate determination of thickness, $t$. The shear band boundary represents a strain field discontinuity. So due to the biasing effects discussed in Sect. 3.1, DIC will represent the spatial transition into the shear band zone as more gradual than it is actually. The result will be a misperception of the location of the shear band boundary (dashed line in Fig. 5b) and hence an overestimation of shear band thickness. The precise magnitude of this overestimation depends on subset size, but also on how far a subset extends physically into the shear band before a difference from the mean field will be registered. To estimate this distance, we took a typical digital image from one of our tests, and using image processing functions within MatLab ${ }^{\circledR}$, rotated the image 60 degrees (a typical shear band inclination), imposed an affine shear deformation of known thickness at a known location, and then rotated the image back to its original orientation. We then performed a DIC analysis between the original and altered images and examined the DIC-determined shear band thickness. The image alteration procedure induces some irreversible image distortion (i.e., while the image rotates, the pixels do not), which alters the grey level values of each pixel; the shear band thickness, however, remains the same. The results indicated that the distance a subset must extend into the shear band to register a differential displacement at its center is about $D_{50}$, as shown in Fig. $5 \mathrm{~b}$ (note, this value is appropriate only for $\beta \approx 60^{\circ}$; for example, as $\beta \rightarrow 0^{\circ}$, the distance becomes infinitesimal).

With this assumption at hand, we proceed to estimate a maximum subset size, $m_{\max }$, as follows. We perform a preliminary DIC analysis using a preliminary subset size, $m_{\text {pre }}$ (say, around $10 \times \mathrm{D}_{50}$ ). From the resulting displacement norm map, a preliminary estimate of shear band thickness, $t_{\text {pre }}$, is then obtained by thresholding the displacement gradient and delineating the portion of the specimen undergoing strain. Then, based on Fig. 5b, the 


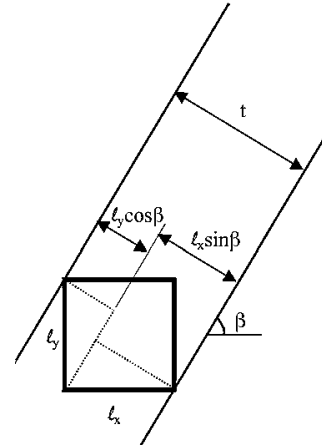

(a)

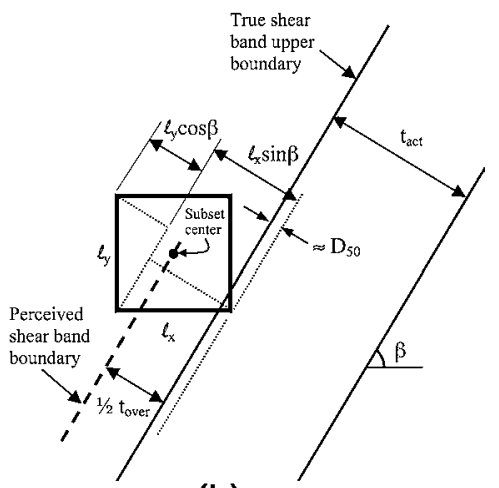

(b)
Fig. 5 DIC subset size considerations: a limiting subset size to fit within shear band of thickness $t$, $\mathbf{b}$ shear band thickness overestimation using DIC

approximate degree to which shear band thickness is overestimated, $t_{\text {over }}$, is calculated by:

$t_{\mathrm{over}}=\left(l_{x} \sin \beta+l_{y} \cos \beta\right)-2 D_{50}$

In Eq. $1, l_{x}=m_{\mathrm{pre}} \cdot x_{\mathrm{s}}$ and $l_{y}=m_{\mathrm{pre}} \cdot y_{\mathrm{s}}, x_{\mathrm{s}}=x$-direction image scale (in $\mathrm{mm} / \mathrm{pixel}$ ), and $y_{\mathrm{s}}=y$-direction image scale (in $\mathrm{mm} / \mathrm{pixel}$ ). The estimated, actual shear band thickness is then determined as $t_{\mathrm{act}}=t_{\mathrm{pre}}-t_{\mathrm{over}}$. Finally, $t_{\text {act }}$ is used to constrain the maximum subset size, $m_{\max }$ :

$m_{\max }=\frac{t_{\mathrm{act}}}{\left(x_{\mathrm{s}} \sin \beta+y_{\mathrm{s}} \cos \beta\right)}$

Note that while $t_{\text {act }}$ is only an estimate of thickness, the image data (discussed below) suggest these values are fairly accurate. These $t_{\text {act }}$ are also used herein for gross shear band shear strain calculations.

To investigate the validity of subset sizes, $m_{\max }$, approximated as above, we look at contours of displacement norm as a function of subset size. Figure 6 shows incremental displacement norm determined for a Concrete sand specimen (test C24) for DIC analyses conducted over a $0.15 \%$ axial strain increment (representing about 5\% gross shear strain, $\gamma$, across the band) for subsets ranging from 25 to 75 pixels, increasing in increments of 10 pixels (the extraneous displacements outside the shear band in the lower and upper middle of the AOI arose from scratches [since repaired] in the acrylic sidewall). Subset center-tocenter spacing is constant at 5 pixels. Horizontal and vertical axes indicate physical position along the AOI in the camera $(x-y)$ basis, the origin of which is at the upper left corner of the image. The same AOI was used in each analysis (as subset size increases, the subset centers get shifted inward, leading to a gradual narrowing of the region of displacement determination). Clearly, as subset size increases, the displacement variation along the shear band changes. For 25- to 55-pixel subset results, five distinct islands of low displacement are captured in each analysis. For both the 25- and 35-pixel results, the sizes and shapes of the islands are more or less the same. However, for 45-pixel subsets and higher, the shapes and sizes of the islands are changing: for example, for 45- and 55-pixel subsets, the 2nd-from-the-bottom low-displacement island is more rounded in shape and smaller in size. For 65- and 75-pixel subsets, islands are either absent or merged, indicating that the distinct, local kinematics within these regions are being diluted amidst the surrounding displacement field. Based on these collective observations, the target length scale below which to obtain distinguishing behavior along the shear band length seems to lie somewhere between 35 and 45 pixels. Using the procedure described above, the limiting subset size, $m_{\max }$, for this specimen was calculated to be 37 pixels. Similar analyses for different tests and different sands yielded similar results. An additional observation from Fig. 6 is that as subset size increases, shear band thickness appears to increase. This serves to validate that shear band thickness overestimation is indeed a function of subset size.

While small subset sizes are desirable to minimize the biasing problem depicted in Fig. 5 and described in Sect. 3.1, subset sizes must also be large enough to reflect an identifiable gray-level variation. We used feature detection methods to investigate the uniformity of "feature" (i.e., distinct sand grains) distribution [35] within our images. Standard deviations of gray levels over small pixel regions (about the size of the largest grains) were calculated. We considered a region to possess a feature if the standard deviation within that region was greater than the standard deviation calculated over the entire image. Once all features were detected as such, we calculated the minimum subset size required for all subsets to capture at least 3 features, thus ensuring they would posses a non-biased, identifiable gray-level pattern [35]. For Fig. 6, subset size was determined to be around 40 pixels, just slightly larger than the subset size dictated by Eq. 2 above. This result helps explain why results for 45-pixel subsets and larger contain much less "noise" than the 25- and 35-pixel subset results. Note, however, that the result from any such analysis depends on the threshold value used to identify a "feature" [26]. Specifically, for our sands, even within regions absent a "feature", gray levels still exhibit some variation (note, for example, the upper left quadrant of Fig. 2c). Note further that during specimen preparation, we had no practical control over the ensuing grain color across the specimen: distinctly colored grains could be uniformly distributed, or clumped together. This illustrates another challenge in DIC use in sands. For these reasons, Eqs. 1 and 2 were used to calculate maximum subset sizes, and then, the lowest subset sizes below this that could still achieve consistent DIC convergence were used. We note 

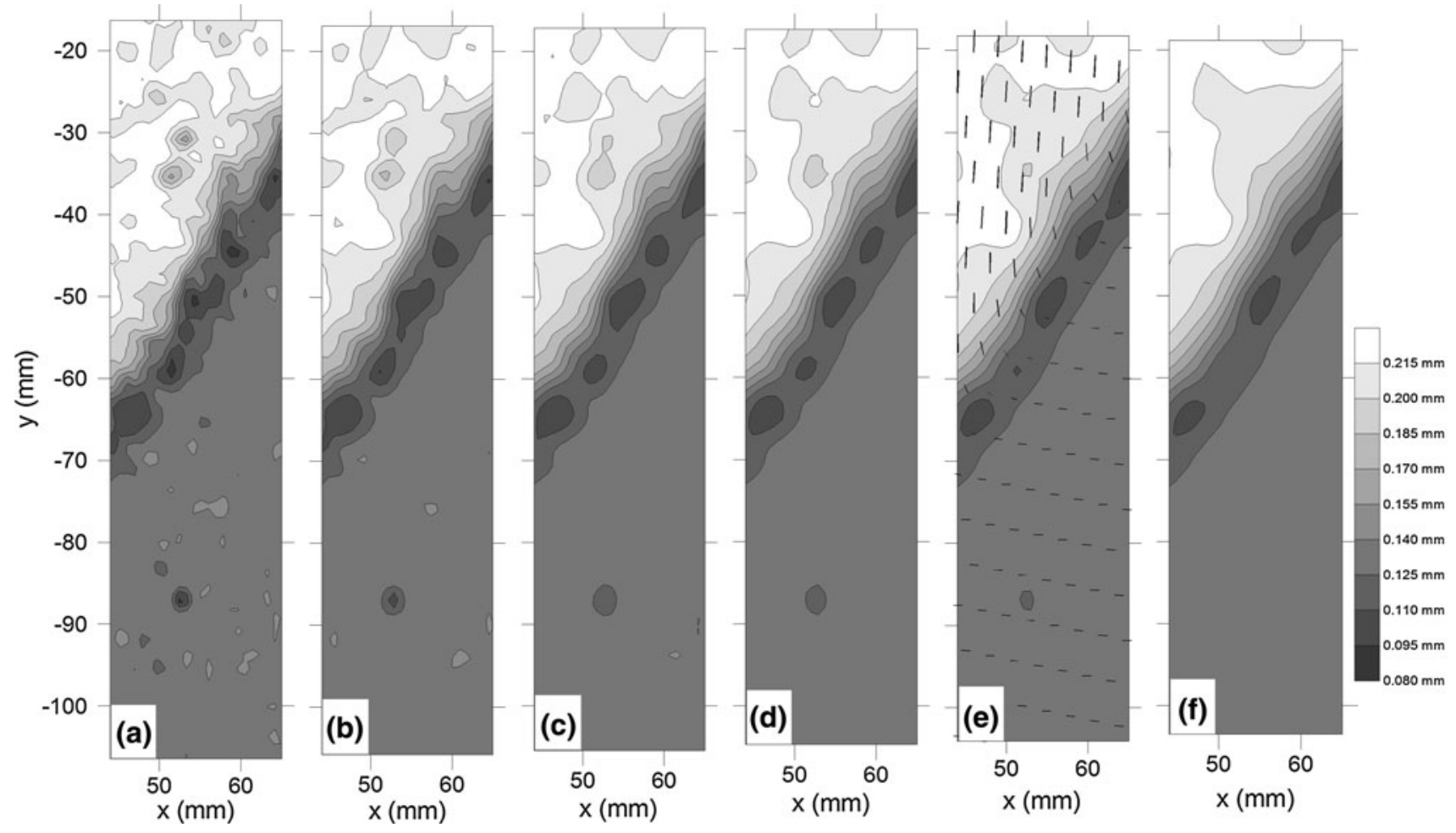

Fig. 6 Displacement norm (camera reference frame) for a Concrete Sand specimen (test C24) as a function of subset size in pixels: a 25, b 35, c 45 , d 55, e 65, f 75. Representative portion of displacement vectors shown only in e for clarity

that subset sizes determined as such are slightly smaller than those utilized previously by the authors [31], but this does not impact the qualitative observations from our previous findings, as will be seen below.

\subsection{Strain increment}

Strain within a narrow shear band is particularly intense, so the strain increment over which DIC analysis are conducted must be carefully chosen to as best as possible accommodate the assumption of affine subset deformation. Here, for our natural sands, it is difficult to quantitatively ascertain if or when deformation is affine (even during the pre-peak test phase when sand deformation is diffuse). However, recall that when a shear band is present, the material outside of the shear band is translating uniformly; thus, subset strain is zero order. By comparing ZNSSD errors within the shear band to those outside the band, we could thus constrain our strain increment to a reasonable level of DIC mapping confidence.

Figure 7 shows contours of ZNSSD error plotted over the correlated portion of an MC sand specimen (test MC 35) for DIC analyses conducted over consecutively larger global axial strain, $\varepsilon_{\mathrm{a}}$, increments, from 0.05 to $0.3 \%$ (approximately corresponding to gross shear band shear strains, $\gamma$, from 1.4 to $8.1 \%$, respectively). Axes indicate physical position along the AOI in the camera $(x-y)$ basis.
We first note that outside the shear band, in the uniformly translating regions, the ZNSSD error measure is unaffected by strain increment (note, ZNSSD $=0$ indicates a perfect match). Inside the shear band, as strain increment increases, as expected, the local ZNSSD error increases. For the $e_{\mathrm{a}}=0.05 \%$ increment $(\gamma \approx 1.4 \%$, Fig. $7 \mathrm{a})$, no recognizable difference in correlation error is seen inside versus outside the shear band (which here extends from the upper left corner of the AOI to about the mid-height of the right side). For the $\varepsilon_{\mathrm{a}}=0.10$ and $0.15 \%$ increments $(\gamma \approx 2.7$ and $4.1 \%$, Figs. $7 \mathrm{~b}$ and $\mathrm{c}$ ), the error magnitudes in some regions within the shear band have increased slightly relative to the uniformly translating material. For the $\varepsilon_{\mathrm{a}}=0.20 \%$ increment $(\gamma \approx 5.4 \%)$ and above (Fig. $7 \mathrm{~d}-\mathrm{f}$ ), the error magnitude along the entire length of the shear band is elevated compared to the regions outside the shear band.

The elevated errors in Fig. 7b-f do not necessarily suggest that subset deformation is non-affine and that DIC reliability is compromised; rather, they merely indicate that subset matching is more challenged compared to conditions of uniform translation. Note from Fig. $7 \mathrm{~b}-\mathrm{f}$ that the spatial locations of the highest errors are consistent along the length of the shear band. Figure $8 \mathrm{a}$ shows for an SC sand specimen (test SC10) contours of DIC correlation error (the Sum of Squared Differences error measure was used in this case) along the length of a shear band, plotted 


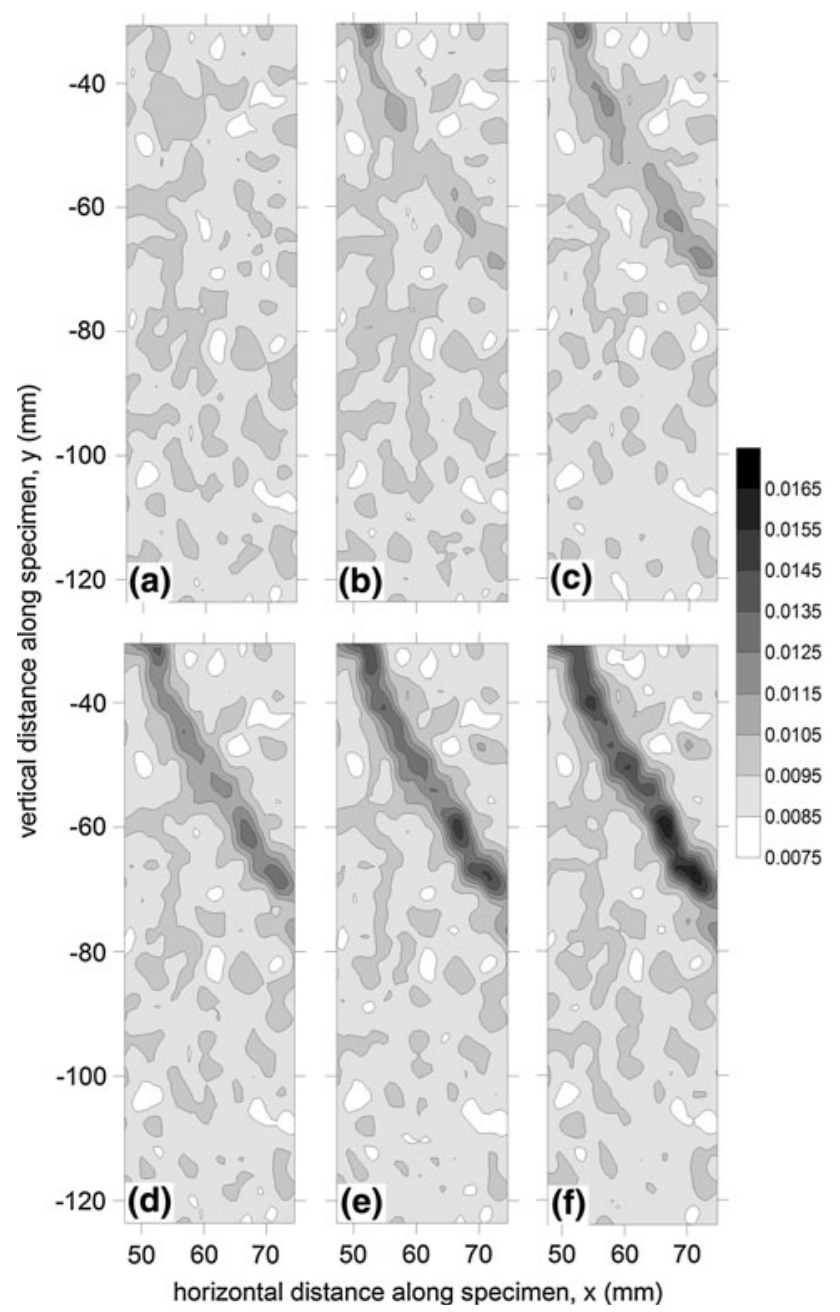

Fig. 7 ZNSSD errors for DIC analyses conducted on an MC sand specimen with a persistent shear band, as a function of increasing axial strain increment: a $0.05 \%$, b $0.10 \%$, c $0.15 \%$, d $0.20 \%$, e $0.25 \%$, and $\mathbf{f} 0.30 \%$

in the rotated, shear band $\left(x^{*}-y^{*}\right)$ basis, with the $x^{*}$-axis oriented parallel to the shear band. Figure $8 \mathrm{~b}$ shows for the same shear band the variation of the spin of the deformation field, $\Omega$, which is calculated from the deformation gradient of the DIC data field (discussed below). A large strain increment of $0.25 \%$ axial strain $(\gamma \approx 11 \%)$ was used to accentuate the observations (note that the larger $\gamma$ for the SC sand compared with the MC sand for the same $\Delta \varepsilon_{\mathrm{a}}$ is reflective of the narrower shear band thickness in the smaller-grained SC sand). We see that regions of high error spatially correlate with regions of high rotation. Thus, the elevated errors appear to be largely caused by significant mesoscale grain-cluster rotation. Recognizing the importance of accurately identifying the high-rotation zones (which, as will be seen below, correlate with vortex structures), the limiting shear strain increment for DIC analysis was taken to be the increment below which the

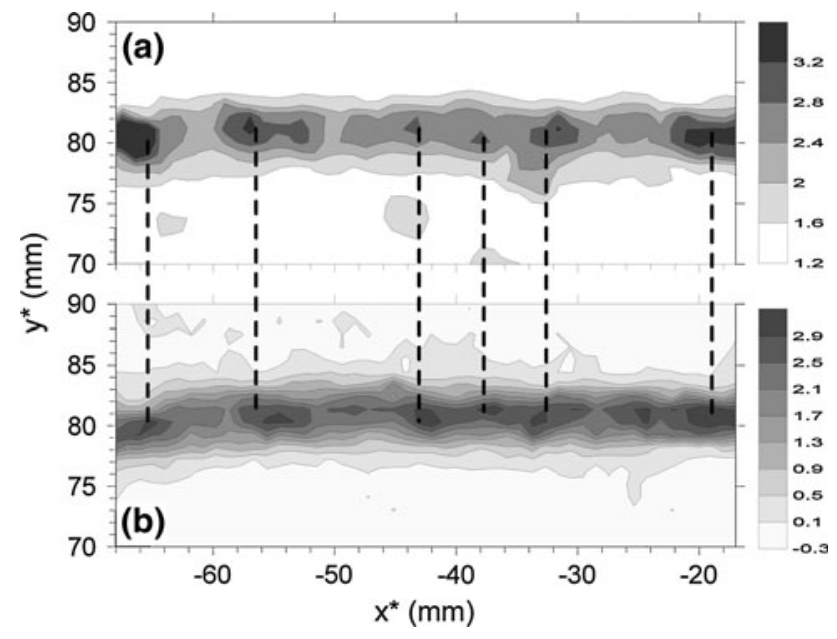

Fig. 8 Variations along the length of a shear band in an SC sand specimen of: a DIC correlation error, and $\mathbf{b}$ kinematic rotation, $\Omega$

ZNSSD errors became uniformly elevated across the entire shear band length: for example, for the specimen depicted in Fig. 7, a limiting axial strain increment of $0.15 \%$ $(\gamma \approx 4.1 \%)$ would be used. Based on these findings, we recommend limiting strain increments for DIC analysis of no more than $4-5 \%$ gross shear band shear strain. To analyze kinematic behavior over larger strain increments, the incremental DIC analyses were accumulated [9].

\section{Strain and kinematic quantities}

The bases for our calculations of kinematic quantities are the displacements of each subset center relative to its neighbors. Thus, continuum-based local strain and kinematic quantities are evaluated from the DIC displacement fields, and we emphasize that non-affine strains calculated from there do NOT reflect individual subset strain. From the rich DIC displacement fields, the local deformation gradient, $\mathbf{F}$, is computed. Local volume changes (assumed to be reflective of in-plane area changes) are then quantified in terms of the Jacobian, J, of $\mathbf{F}$. Macro-rotations, $\Omega$, that correspond to rigid body rotations are obtained from the polar decomposition of $\mathbf{F}$. Precise formulations for these quantities are described in detail in Chupin et al. [9].

In most cases, the strain increment needed to capture the pronounced kinematic signatures of force chain buckling or the full coherency of vortex structures was larger than the strain increments afforded by the DIC technique. Thus, to evaluate behavior over wider strain increments, the incremental, Eulerian-based DIC results were accumulated in a Lagrangian frame [9]. Then, the deformation gradients were computed over the accumulated displacement field, and strain and kinematic measures subsequently determined. 
As explained by Williams and Rege [48], coherent vortex-like structures, or "circulation cells", represent deviations of the granular flow fields from the continuum field. Thus, to examine these "residual displacements," the contribution of the mean deformation field must be subtracted from the DIC-measured displacements. While the shear band boundaries were roughly linear in an average sense (Fig. 4), locally, due to innate grain heterogeneity, the shear band boundaries were nonlinear. Thus, to calculate the residual fields, we first transform the measured displacements into the stationary sled configuration (Fig. 4b). The local displacement gradient is calculated, and the lower and upper boundaries of shear band are found by thresholding the displacement gradient. Then, due to the local variations in shear band thickness, for each column in the DIC data grid, we individually superimpose an affine, first-order shear displacement field inside the shear band. This mean displacement field is then subtracted from the observed displacements to obtain the residual displacement field.

\section{Results}

\subsection{Mesoscale instabilities and vortices inside shear bands}

We demonstrate the nature of non-affine, mesoscale shear band deformation through analysis of data from the MC sand test specimen (test MC24) shown in Figs. 9 and 10. The global stress and volumetric strain data for this test are shown in Fig. 9 (note that the slight volumetric contraction seen near the end of the test was due to a leak that formed in the drainage line; the "bumps" in the curves at about $2 \%$ axial strain were due to an error in the data acquisition system; and the "bump" in the shear stress curve just after peak stress was due to a momentary fluctuation in cell pressure). Figure 10 shows the spatial variations of kinematic quantities and residual displacements along the length of the shear band at the global softening-critical state transition, over the axial strain increment $7.64-8.26 \%$
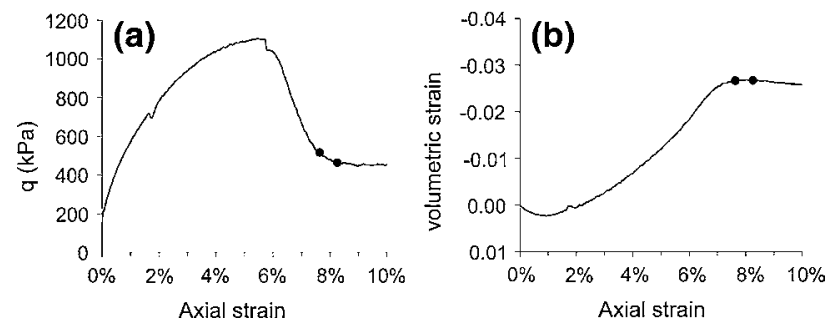

Fig. 9 Global shear stress (a) and volumetric strain (b) versus global axial strain for plane strain compression test on dense MC sand. Data points indicate the analysis increment of figure
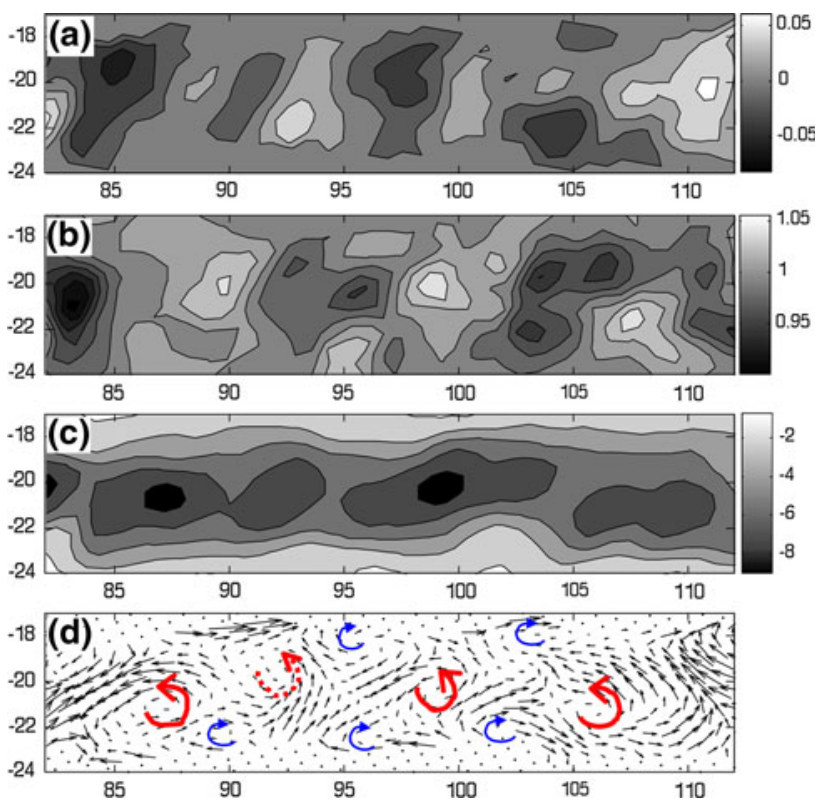

Fig. 10 Kinematics and residual displacements along shear band of specimen MC24: a $v^{*}(\mathrm{~mm})$; b Jacobian, $J$; $\mathbf{c}$ rotation, $\Omega$; $\mathbf{d}$ residual displacement vectors. Sense of shear is top of band moving left, bottom stationary

(indicated by the data points on Fig. 9), which represents roughly $\gamma \approx 18 \%$ across the shear band. The kinematics were calculated from displacements accumulated over six consecutive, incremental DIC analyses over $0.1 \%$ axial strain increments $(\gamma \approx 3 \%)$. Subsets were 63 pixels square $\left(m_{\max } \approx 73\right.$ pixels), spaced 11 pixels on center. Horizontal and vertical axes indicate physical dimensions (in millimeters) along and across the shear band (shear band basis, $\left.x^{*}-y^{*}\right)$. Sense of shear is the top of the shear band moving left, bottom is stationary (i.e., sled reference frame). Figure 10a shows contours of the displacement component perpendicular to the shear band axis, $v^{*}$. Fig. 10b shows contours of the Jacobian, J, along the shear band length, where $\mathrm{J}>1$ indicates volumetric dilation (expansion) and $\mathrm{J}<1$ indicates volumetric contraction. Figure $10 \mathrm{c}$ shows kinematic rotation, $\Omega$, where $\mathrm{CCW}$ rotation is negative.

As in previous works [31], a systematic, spatial pattern in the kinematics is seen: in the direction of shear, peaks (absolute value) in rotation, $\Omega$ (Fig. 10c), are followed by downward perpendicular displacement $\left(v^{*}<1\right.$, or darkest grays in Fig. 10a), and volumetric contraction $(\mathrm{J}<1$, or darkest grays in Fig. 10b), followed by neutral or upward $v^{*}$ (white and light to medium grays, Fig. 10a) and volumetric dilation ( $\mathrm{J}>1$, or white to light grays, Fig. 10b), which are again followed by high rotation, $\Omega$, and so on. Rechenmacher et al. [31] argued that this pattern was indicative of a collective, coordinated force chain collapse event. Note that this pattern first emerges at the softening/ 
critical state transition. The temporal evolution of the pattern is discussed in Rechenmacher et al. [31].

The primary justification that the above pattern is indicative of actual force chain collapse is based on similar kinematics seen from numerical simulations, e.g., [17, 24, 42, 43], in which loss of particle contacts, particle force changes, and/ or internal energy changes associated with force chain collapse were quantitatively measured. Here, we show that the kinematical patterns tie in with non-affine deformation in the form of vortex structures. Figure 10d shows vectors of residual displacement, the lengths of which have been scaled relative to their respective magnitude. Note that if shear within the shear band was affine, then residual displacements would be zero. Clearly, deformation along the shear band is almost entirely non-affine. Along the length of the shear band, we identify three coherent vortex-like structures, characterized by displacement vectors oriented in a rigid-body-like rotational sense (i.e., [49]), and indicated in Fig. 10d by the thick, counter-clockwise-directed arrows (note, a fourth circulating structure is seen at $x^{*} \approx 92$, but as it is only semiformed, we identify it with a dotted arrow). The spatial locations of these vortex-like structures line up nearly exactly with the peaks (absolute value) in rotational strain (Fig. 10c; the weakness of the "peak" at $x^{*} \approx 92$ reflects the semiformed nature of the circulating structure at this location). At the conflux between adjacent vortex structures, local displacements are in opposition and volumetric strain is contractive (Fig. 10b). Tordesillas et al. [43] saw "microbands" of slip at these junctures. Due to the subset biasing effect discussed in Sect. 3.1, microbands in our data would be reflected in a finite-sized spatial zone of parallel displacement vectors that gradually transition to opposite orientations. In Fig. 10d, we do not see evidence of such structures, suggesting that slip deformation may not be a primary mode of dissipation in the subrounded- to subangular-shaped sands tested here. A further observation in Fig. 10d is the presence of opposite-trending rotational entities near the shear band boundaries, in between the main shear band vortices, indicated by thinner, clockwise arrows nearer to the shear band boundaries. These rotational "wakes" have not been observed in numerical simulations on circular particles $[1,48]$. We emphasize that the observations above apply for subrounded to subangular particles and that the potential effects of particle elongation on vortex structure formation and patterning are beyond the scope of this study.

To validate that the vortex structures seen in Fig. 10 are representative of actual mesoscale motion, Fig. 11 shows a close up of a portion of the shear band of Fig. 10 corresponding to $x^{*} \approx 95-105$. Figure 11a shows grain positions corresponding to the image collected at the beginning of the accumulated increment represented in Figs. 10, and 11b shows roughly the same grains for the final image of the increment (sense of shear is the top moving horizontally left,

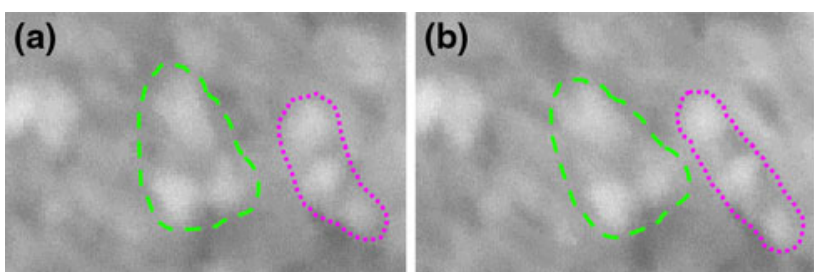

Fig. 11 Close-up of a portion of the shear band of Fig. 9 centered around $x^{*} \approx 101$ : a pre-vortex formation; b post-vortex formation. Sense of shear is top of shear band translating horizontally left, bottom of shear band translating horizontally right

and the bottom moving horizontally right). We focus first on the three prominent, white sand grains arranged in a triangular position in the center of Fig. 11a. The three sand grains are seen to have collectively rotated during this increment. The center of these grains corresponds approximately to $x^{*} \approx 102$, which in Fig. 10d is on the right-hand side of the center-most vortex structure. Thus, this meso-scale graincluster rotation appears to have been precisely captured by DIC. We additionally examine the three white, diagonally arranged grains on the right side of Fig. 11a, b. From Fig. 11a to b, the top-most of these three grains has translated leftward and is seen to almost touch the rightmost of the three previously referenced, triangularly arranged grains. The meeting point of these two grains corresponds to the region of converging vectors located at approximately $x^{*} \approx 104$ in Fig. 10d and the associated zone of volumetric contraction in Fig. 10b. Moreover, all three grains have stretched apart, and this behavior corresponds to the dilative zone seen at $x^{*} \approx 106$ in Fig. 10b. This correspondence between actual grain motion and DIC-derived kinematics serves to highlight the promise the DIC technique holds toward availing further understanding of meso-scale sand behavior and in particular the ties among micro-, meso-, and macro-scale responses (e.g., [4]). Current analyses are focused on tracking the fate of the vortex structures, and non-affine deformation in general, further into the critical state.

\subsection{Shear band thickness}

Note that the thickness of the zone of active kinematics within the shear band of Fig. 10 is wider than the actual thickness due to the inherent overestimation discussed in Sect. 3.2. Using the procedure described in Sect. 3.2, we calculated adjusted estimates of actual shear band thicknesses for several of our MC and SC sand tests. These calculations indicate that for the MC sand, shear band thickness, $t_{\text {act }}$, was around (6-7.5) $\times D_{50}$. For the SC sand, $t_{\text {act }}$ was $(8-9) \times D_{50}$. Interestingly, these values fairly closely agree with the recent theoretical determination of $8 \times D_{50}$ by Tordesillas et al. [44] as the critical length scale for shear band thickness and force chain stability based on an elastic buckling analysis. The difference in 
thickness between MC and SC sand may be due in part to subtle differences in grain shape and angularity. A more rigorous experimental analysis of the effects of grain shape on shear band thickness is underway.

Taking advantage of the digital format of observation in our experiments and the frequent imaging, we could compile movies of grain deformation. From such movies, we can attempt to qualitatively view shear band deformation and gage the approximate number of grains actively involved in shear band deformation. Such an exercise was conducted for the MC sand test represented in Figs. 10 and 11. Approximately six to seven grains were seen to comprise the shear band. Performing the calculations of Sect. 3.2, shear band thickness, $t_{\text {act }}$, was evaluated to be $6.3 \times D_{50}$. The agreement lends validity to the assumptions underlying Eqs. 1 and 2, and our estimates of thickness quoted above.

Additionally, we note that the thickness values reported by Rechenmacher and Finno [30] determined from DIC for the same unsieved, well-graded Concrete sand as above (Table 1), as well as sieved, uniform Masonry sand $\left(D_{50}=0.32 \mathrm{~mm}, C_{\mathrm{u}}=1.3, C_{\mathrm{c}}=1.02, G_{\mathrm{s}}=2.68\right)$, were determined without consideration of the effect of subset size and thus represent over-estimates of thickness. They reported average shear band thickness for the Masonry sand to be $17.5 \times D_{50}$, and for Concrete sand, $16 \times D_{50}$. Applying the procedure of Sect. 3.2, the revised estimates are: (7-9) $\times D_{50}$ for Mason sand and (8-9.5) $\times D_{50}$ for Concrete sand. Note that the sands tested by Rechenmacher and Finno [30] represented a variety of initial states (bulk relative densities between 49 and 84\% [28] and effective confining pressures ranging from 100 to just over $400 \mathrm{kPa}$ ). These sands also varied in grain shape and grain size distribution. Note also, as these represented first-generation tests using DIC, and the DIC analyses performed over a variety of different strain increments, many of which were much larger than suggested above. Even so, the values are in the same range as the $\mathrm{MC}$ and $\mathrm{SC}$ sands tested herein. While more data are needed to constrain the influences of grain shape, grain size distribution and soil state on shear band thickness, these revised estimates, we feel, offer more accurate representations of shear band thickness, in particular in light of the agreement with image data described above.

\section{Conclusions}

Digital Image Correlation (DIC) has been employed to evaluate and characterize meso-scale, non-affine deformations, and kinematics associated with granular instability in the form of shear bands in sands developed during plane strain compression. We have performed a rigorous assessment of DIC analysis parameters and provide updated recommendations from previous research for DIC subset sizes and limiting strain increments for optimal characterization of meso-scale displacements within and around shear bands, which should provide guidance for others using this technique. DIC has been used to characterize local, grain-scale shear band displacements, and kinematics within shear bands in subrounded to subangular sands. Systematic, spatial fluctuations in kinematical quantities along the shear band length that in previous research were tied to force chain buckling were here seen to correlate with the presence of coherent vortex structures or "circulation cells".

We have devised a means to systematically compensate for a biasing effect in DIC due to non-affine deformation, such as at the shear band boundary, to enable estimations of shear band thickness from DIC data. Actual shear band thickness was estimated for the three sands tested here, plus the data for the sands tested previously by the author [30] were re-analyzed to accommodate the effect of subset size. In all cases, shear band thickness ranged between 6 and 9.5 times $D_{50}$. Agreement with image data and a recent theoretical finding serve to validate these estimates. The spread in these results indicates that grain shape, angularity, and grain size distribution may also affect observed shear band thickness. Regardless, the DIC technique described here can play an integral role in efforts to discern these effects and in ongoing research efforts to understand the tie between micro- and macroscopic sand response.

Acknowledgments The authors thank the National Science Foundation (Grant CMMI-0748284) and the University of Southern California for their financial support of this research. We also sincerely appreciate the kind advice of Dr. Hubert Schreier. We additionally thank the anonymous reviewers for their keen suggestions in helping to strengthen this manuscript.

\section{References}

1. Alonso-Marroquin F, Vardoulakis I, Herrmann HJ, Weatherley D, Mora P (2006) Effect of rolling on dissipation in fault gouges. Phys Rev E 74:031306

2. Alshibli KA, Hasan A (2008) Spatial variation of void ratio and shear band thickness in sand using X-ray computed tomography. Geotechnique 58(4):249-257

3. Alshibli KA, Sture S (1999) Sand shear band thickness measurements by digital imaging techniques. J Comput Civil Eng 13(2):103-109

4. Andrade JE, Avila CF, Hall SA, Lenoir N, Viggiani G (2011) Multiscale modeling and characterization of granular matter: from grain kinematics to continuum mechanics. J Mech Phys Solids 59:237-250

5. Bardet JP, Proubet J (1991) A numerical investigation of the structure of persistent shear bands in granular media. Geotechnique 41(4):599-613

6. Bonert M, Brémand F, Doumalin P, Dupré J-C, Fazzini M, Grédiac M, Hild F, Mistou S, Jolimard J, Orteu J-J, Robert L, Surrel Y, Vacher P, Wattrisse B (2009) Assessment of digital 
image correlation measurement errors: methodology and results. Exp Mech 49:353-370

7. Borja RI, Andrade JE (2006) Critical state plasticity. Part VI: Meso-scale finite element simulation of strain localization in discrete granular materials. Comput Methods Appl Mech Eng 195(37-40):5115-5140

8. Bruck HA, McNeill SR, Sutton MA, Peters WH III (1989) Digital image correlation using Newton-Raphson method of partial differential corrections. Exp Mech 29(3):261-268

9. Chupin O, Rechenmacher AL, Abedi S (2011) Finite strain analysis of nonuniform deformations inside shear bands in sands. Int J Numer Anal Methods Geomech (in press)

10. Daniels KE, Hayman NW (2008) Force chains in seismogenic faults visualized with photelastic granular shear experiments. J Geophys Res 13:B11411

11. Drescher A, Josselin De, de Jong G (1972) Photoelastic verification of a mechanical model for the flow of a granular material. J Mech Phys Solids 20:337-351

12. Evans TM, Frost JD (2010) Multiscale investigation of shear bands in sand: physical and numerical experiments. Int $\mathrm{J}$ Numer Anal Methods Geomech 34(15):1634-1650

13. Finno RJ, Rechenmacher AL (2003) Effects of consolidation history on critical state of sand. J Geotech Geoenviron Eng 129(4):350-360

14. Finno RJ, Harris WW, Mooney MA, Viggiani G (1997) Shear bands in plane strain compression of loose sand. Géotechnique 47(1):149-165

15. Hasan A, Alshibli KA (2010) Experimental assessment of 3D particle-to-particle interaction within sheared sand using synchrotron microtomography. Geotechnique 60(5):369-379

16. Howell DW, Behringer RP, Veje CT (1999) Fluctuations in granular media. Chaos 9(3):559-572

17. Iwashita K, Oda M (2000) Micro-deformation mechanisms of shear banding process based on modified distinct element method. Powder Technol 109(1-3):192-205

18. Jang DJ, Frost JD (2000) Use of image analysis to study the microstructure of a failed sand specimen. Can Geotech $\mathbf{J}$ 37:1141-1149

19. Knauss WG, Chasiotis I, Huang Y (2003) Mechanical measurements at the micron and nanometer scales. Mech Mater 35:217-231

20. Lesniewska D, Wood DM (2009) Observations of stresses and strains in a granular material. J Eng Mech 135(9):1038-1054

21. Liang L, Saada A, Figueroa JL, Cope CT (1997) The use of digital image processing in monitoring shear band development. Geotech Test J 20(3):324-339

22. Majmudar TS, Behringer RP (2005) Contact force measurements and stress-induced anisotropy in granular materials. Nature 435:1079-1082

23. Mooney MA (1996) An experimental study of strain localization and the mechanical behavior of sand. PhD Thesis, Northwestern University, Evanston, IL

24. Oda M, Iwashita K (2000) Study on couple stress and shear band development in granular media based on numerical simulation analysis. Int J Eng Sci 38(15):1713-1740

25. Oda M, Kazama H (1998) Microstructure of shear bands and its relation to the mechanisms of dilatancy and failure of dense granular soils. Geotechnique 48(4):465-481

26. Pan B, Xie H, Wang Z, Qian K, Wang Z (2008) Study on subset size selection in digital image correlation for speckle patterns. Opt Express 16(10):7037-7048

27. Poissant J, Barthelat F (2010) A novel "Subset Splitting" procedure for digital image correlation on discontinuous displacement fields. Exp Mech 50:353-364

28. Rechenmacher AL (2000) Effects of consolidation history and shear rate on the critical state of two sands. PhD. Thesis, Northwestern University, Evanston, IL
29. Rechenmacher AL (2006) Grain-scale processes governing shear band initiation and evolution in sands. J Mech Phys Solids $54: 22-45$

30. Rechenmacher AL, Finno RJ (2004) Digital image correlation to evaluate shear banding in dilative sands. Geotech Test J 27(1):13-22

31. Rechenmacher A, Abedi S, Chupin O (2010) Evolution of force chains in Shear Bands in sand. Geotechnique 60(5):343-351

32. Réthoré J, Hild F, Roux S (2007) Shear-band capturing using a multiscale extended digital image correlation technique. Comput Methods Appl Mech Eng 196(49-52):5016-5030

33. Röchter L, Köning D, Schanz T, Triantafyllidis T (2010) Shear banding and strain softening in plane strain extension: physical modeling. Granular Matter 12(3):287-301

34. Sadrekarimi A, Olson SM (2010) Shear Band Formation Observed in Ring Shear Tests on Sandy Soils. J Geotech Geoenviron Eng 136(2):366-375

35. Schreier HW, Sutton MA (2002) Systematic errors in digital image correlation due to undermatched subset shape functions. Exp Mech 42(3):303-310

36. Slominski C, Niedostatkiewicz N, Tejchman J (2007) Application of particle image velocimetry (PIV) for deformation measurement during granular silo flow. Powder Technol 173:1-18

37. Sutton MA, Wolters WJ, Peters WH, Ranson WF, McNeill SR (1983) Determination of displacements using an improved digital image correlation method. Image Vision Comput 1(3):133-139

38. Sutton MA, McNeill SR, Helm JD, Chao YJ (2000) Advances in two-dimensional and three-dimensional computer vision. In: Rastogi PK (ed) Photomechanics: topics in applied physics. Springer, Berlin, pp 323-372

39. Sutton MA, Orteau J-J, Schreier HW (2009) Image correlation for shape, motion and deformation measurements: basic concepts, theory and applications. Springer, New York

40. Tagliaferri F, Waller J, Andò E, Hall SA, Viggiani G, Bésuelle P, DeJong JT (2011) Observing strain localization processes in biocemented sand using X-ray imaging. Granular Matter 13:247-250

41. Thornton C, Zhang L (2006) A numerical examination of shear banding and simple shear non-coaxial flow rules. Philsophic Mag 86(20-21):3425-3452

42. Tordesillas A, Muthuswamy M (2009) On the modeling of confined buckling of force chains. J Mech Phys Solids 57(4):706-727

43. Tordesillas A, Muthuswamy M, Walsh SDC (2008) Mesoscale measures of nonaffine deformation in dense granular assemblies. J Eng Mech 134(12):1095-1113

44. Tordesillas A, Hunt G, Shi J (2011) A characteristic length scale in confined elastic buckling of a force chain. Granular Matter 13:215-218

45. Utter B, Behringer RP (2004) Self-diffusion in dense granular shear flows. Phys Rev E 69:031308

46. Walsh SDC, Tordesillias A, Peters JF (2007) Development of micromechanical models for granular media. Granular Matter 9(5):337-352

47. Wang Y, Cuitiño AM (2002) Full-field measurements of heterogeneous deformation patterns on polymeric foams using digital image correlation. Int J Solids Struct 39:3777-3796

48. Williams JR, Rege N (1997) Coherent vortex structures in deforming granular materials. Mech Cohesive Friction Mater 2(3):223-236

49. Williams JR, Rege N (1997) The development of circulation cell structures in granular materials undergoing compression. Powder Technol 90:187-194

50. Wolf H, König D, Triantafyllidis T (2006) The influence of the stress-strain behavior of non-cohesive soils on the geometry of shear band systems under extensional strain. Eng Struct 28:1760-1773 